Strahlenther Onkol 2013 · 189:907-907

DOI 10.1007/s00066-013-0426-y

Online publiziert: 22. August 2013

(c) Springer-Verlag Berlin Heidelberg 2013

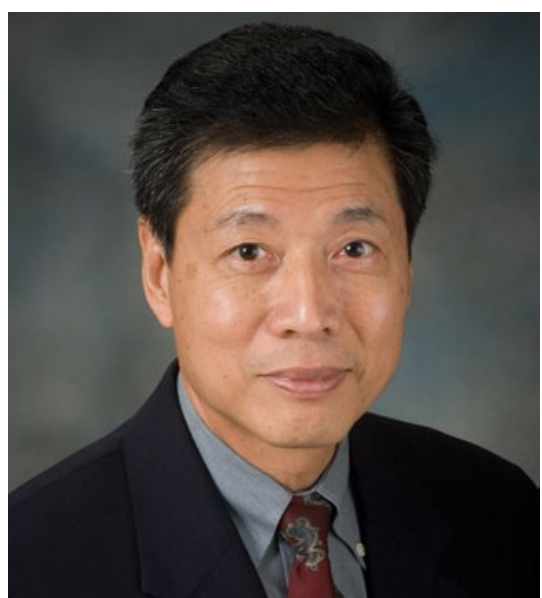

$\Delta$ Prof. Dr. Kian Ang

(Mit freundlicher Genehmigung)

Kian Ang starb am 19. Juni 2013 im Alter von 63 Jahren im Kreise seiner Familie in Houston, Texas. Nur wenige Tage vorher war eine fortgeschrittene Krebserkrankung diagnostiziert worden. Kian Ang war einer der weltweit führenden Radioonkologen und Krebsforscher und seit 2008 Ehrenmitglied der DEGRO.

Kian Ang wurde in China geboren und wuchs in Indonesien auf, bevor er Medizin an der Katholischen Universität in Leuven, Belgien, studierte. Dort absolvierte er auch seine Weiterbildung zum Radioonkologen und promovierte zum $\mathrm{Ph}$. D. Im Anschluss an die Weiterbildung folgte er einem Ruf an das MD Anderson Cancer Center der University of Texas in Houston, wo er die letzten 29 Jahre als Kliniker und Forscher arbeitete. Zuletzt hatte er den Gilbert-Fletcher-DistinguishedMemorial-Lehrstuhl inne.

Kian Ang war zweifelsohne über die letzten Jahrzehnte der international führende Radioonkologe auf dem Gebiet der Kopf-Hals-Tumoren. Hier hat er bahnbrechende klinische Studien initiiert, die von

\title{
M. Baumann
}

Klinik für Strahlentherapie und Radioonkologie und OncoRay-Zentrum für Medizinische Strahlenforschung und Onkologie, Universitätsklinikum Carl Gustav Carus an der Technischen Universität Dresden

\section{Zum Gedenken an Prof. Dr. Kian Ang}

der Optimierung von Dosis und Fraktionierungskonzepten über die Radiochemotherapie bis zur Einführung der ersten molekularen, wirksamen Substanz, Cetuximab, in die Radioonkologie reichten. Als herausragender Kliniker und Lehrer ist er auch der Herausgeber des führenden Lehrbuches der modernen Behandlung von Kopf-Hals-Tumoren. Von besonderer Bedeutung für seine klinische Tätigkeit waren die Forschungsaktivitäten im strahlenbiologischen Labor, die Kian Ang über seine gesamte Karriere hinweg verfolgte. Hiermit wurde er zum Vorbild für zwei komplette Generationen von translational ausgerichteten „Clinician Scientists" in unserem Fach. Fest mit seinem Namen verbunden sind unsere heutigen Kenntnisse zur Strahlentoleranz und Erholungsfähigkeit des Rückenmarks, der Nachweis der relativen Strahlenempfindlichkeit HPV-positiver Plattenepithelkarzinome des Kopf-Hals-Bereichs und ein großes Spektrum von Untersuchungen zum spezifischen Einsatz molekular gerichteter Substanzen in Kombination mit Bestrahlung. Für seine herausragenden Leistungen in der radioonkologischen Forschung wurde Kian Ang mit den wichtigsten Wissenschaftspreisen unseres Fachgebiets, darunter die RegaudGold-Medal der ESTRO und die ASTROGold-Medal, ausgezeichnet.

Zur europäischen und deutschen Radioonkologie hatte Kian Ang auch aus den USA heraus ausgesprochen enge und freundschaftliche Bindungen. Er war regelmäßiger Sprecher auf Kongressen in Europa und begeisterte seine Zuhörer mit brillanten Vorträgen, die mit neuesten Daten gespickt waren. Als Mitglied einer Reihe wissenschaftlicher Beratungsgremien war es häufig Kian Ang, der mit seiner Reputation Vertreter anderer onkologischer Diszi- plinen und Drittmittelgeber davon überzeugen konnte, wie wesentlich die radioonkologische Forschung im Kontext der allgemeinen Krebsforschung ist. Viele junge Strahlentherapeuten und Strahlenbiologen bekamen durch Einladung von Kian Ang die Gelegenheit am MD Anderson Cancer Center klinische Hospitationen und Forschungsaufenthalte durchzuführen - stimulierende Eindrücke die später an den Heimatinstitutionen Forschung und klinische Tätigkeit enorm bereicherten. Es ist sicher nicht übertrieben, zu sagen, dass Kian Ang eine tragende Brücke der Radioonkologie über den Atlantik war.

Für viele von uns war Kian Ang aber nicht nur ein herausragender Vertreter unseres Fachs, sondern auch ein enger persönlicher Freund mit viel Witz, Charme, Verbindlichkeit und ausgeprägter Bodenhaftung. Er liebte Reisen zusammen mit seinen Freunden, Tennis, schnelle Autos, Musik und abendliche Unterhaltungen bei einem guten Glas Wein.

Kian Ang hinterlässt seine Ehefrau Sunny und seine Kinder Angelica mit Ramzi und Dimitri mit Kimberly. Die DEGRO trauert mit seiner lieben Familie. Wir werden Kian als einen großen Radioonkologen, Förderer und Freund immer in dankbarer Erinnerung behalten.

Michael Baumann, Dresden, Volker Budach, Berlin, und Michael Molls, München

\section{Korrespondenzadresse}

Prof. Dr. M. Baumann

Klinik für Strahlentherapie und Radioonkologie und OncoRay-Zentrum für Medizinische

Strahlenforschung und Onkologie,

Universitätsklinikum Carl Gustav Carus an der

Technischen Universität Dresden

Fetscherstr. 74, 01307 Dresden

Michael.Baumann@uniklinikum-dresden.de 